\title{
Pedagogic Exploration in Adapting Automatic Writing Evaluation Software into University Writing Classes
}

\author{
Wei-Yan Li, Kevin Kau \& Chih-Jou Hu \\ National Taiwan University, Taiwan
}

\begin{abstract}
Automated Writing Evaluation (AWE) is computer-generated scoring and feedback used to evaluate and provide feedback on writing, and it is sometimes used for both assessment and instructional purposes. While it has relatively high precision for some types of writing errors, recall is much lower than feedback provided by a trained instructor. Despite this, AWE has the possibility of playing an important role, specifically, in supplementing teacher feedback to reinforce the learning process of students as well as to alleviate writing teachers' stress. However, limited research has been published on the ways AWE could be integrated as an instructional tool, especially in big-sized writing classes at higher education. Therefore, our study aimed to investigate whether the integration of AWE in big-sized English academic writing classes could enhance teaching and learning. To achieve this, both quantitative and qualitative data were collected from two AWE-assisted writing classes in 2020. Consistent with some previous studies conducted in smaller classes, the results of 163 students' essays comparisons showed that Grammarly enabled most students (71\%) to become more aware of some specific writing problems, namely, the issue of formality and grammatical, lexical and sentence structure errors. However, the interview data of 40 students revealed that most students $(n=34)$ considered Grammarly most useful at the post-writing stage but a distraction at outlining and writing stages. Lastly, the two teacher participants suggested the value of AWE in facilitating the quality of peer reviews, as it effectively provided guidance for discussion and motivated the students to negotiate for meaning.
\end{abstract}

Keywords: instructional tool; academic writing; big-sized classes; peer review; writing feedback 